Review

\title{
COVID 19-How is the Exposure of the Pediatric Population? A Systematic Review Protocol
}

\author{
${ }^{1}$ Vitória Monteiro Monte Oliveira, ${ }^{2}$ Paulo Francisco de Almeida-Neto, ${ }^{1}$ Francisca Dalila Paiva Damasceno \\ de Lima, ${ }^{1}$ Stefanny Karla Ferreira de Sousa, ${ }^{1}$ André Carvalho de Sousa, ${ }^{1}$ Ruth Silva Galdino, ${ }^{3}$ Dihogo \\ Gama de Matos, ${ }^{2}$ Breno Guilherme de Araújo Tinôco Cabral and ${ }^{1}$ Glaucia Posso Lima \\ ${ }^{1}$ Health Sciences Center-Ceará State University, UECE, Fortaleza, CE, Brazil \\ ${ }^{2}$ Health Sciences Center, Department of Physical Education-Federal University of Rio Grande do Norte, UFRN, Natal, RN, Brazil \\ ${ }^{3}$ Group of Studies and Research of Performance, Sport, Health and Paralympic Sports-GEPEPS, \\ The Federal University of Sergipe, UFS-São Cristovão, Sergipe, Brazil
}

\author{
Article history \\ Received: 03-01-2021 \\ Revised: 24-03-2021 \\ Accepted: 27-03-2021 \\ Corresponding Author: \\ Vitória Monteiro Monte \\ Oliveira \\ Health Sciences Center-Ceará \\ State University, UECE, \\ Fortaleza, CE, Brazil \\ Email: vitoria.monte@ aluno.uece.br
}

\begin{abstract}
Solid information on exposure and how to treat COVID-19 in pediatric patients is not yet conclusive in the literature, because of this it is necessary to make a critical analysis of the available data in order to provide common points on the theme in question. This systematic review protocol is registered in PROSPERO (ID: CRD42020181835) and aims through searches in the Databases PubMed, Google Scholar, LILACS and CINAHL, to group the studies published with the theme COVID-19 and pediatric between 2019 and 2020. The MeSH descriptors: COVID-19, child and children will be used in English and Chinese. Observational research, clinical trials and literature reviews will be included, where the study sample was composed of pediatric patients of both sexes aged between 6 and 17 years. The Rayyan QCRI tool® will be used to manage revision data. The analysis of the feasibilities of observational studies will be done by the Loney scale, clinical trials by the Cocharne scale and review studies by the Overview Quality Assessment Questionnaire (OQAQ). The authors intend that the study is completed in the second semester of 2021. The choice of the theme is justified because the results of the future research are useful for the elaboration of public health measures that offer the promotion of effective treatment during the recovery of pediatric patients infected with COVID-19.
\end{abstract}

Keywords: COVID-19, Child, Children, Systematic Review

\section{Introduction}

\section{Background}

\section{Description of Condition and Setting}

At the end of the last four months of 2019, the government of China warned the World Health Organization (WHO) about cases of unknown pneumonia that was infecting hundreds of citizens in the city of Wuhan, Hubei province, Central China (WHO, 2019). Over time, scientists pointed out that the pathogen was an acute high-severity respiratory syndrome, which occurred due to contamination by the SARS-VOC-2 virus (Gorbalenya et al., 2020). SARS-COV-2 became popularly known as COVID-19, the virus belongs to the taxonomic subfamily Orthocoronavirinae of the family Coronaviridae, of the order Nidovirales and is characterized by genetic structures that have a simple positive sense RNA-based genome (Payne, 2017; Duarte, 2020; Li et al., 2020).

There is still no cure or treatment for COVID-19, however it is known that its contamination rate is high and that precautionary measures must be taken, such as social distance, the use of masks and constant body hygiene (WHO, 2019; Gorbalenya et al., 2020; Payne, 2017; Duarte, 2020; Li et al., 2020; Sohrabi et al., 2020). It is known that COVID-19 causes clots (i.e., thrombi) in the respiratory system, which make it difficult to transport oxygen. 5-6 In this sense, individuals of any age group who have weaknesses in the vascular and/or 
respiratory systems need care special cases, as is the case of subjects suffering from metabolic diseases, heart disease, obese and elderly (Sohrabi et al., 2020).

However, it appears that COVID-19 affects the pediatric population on a less intensive scale (Zimmermann and Curtis, 2020). Preliminary evidence indicates that young pediatric patients may be infected and not point out symptoms of the disease, which is of concern to health authorities around the world (WHO, 2019; Gorbalenya et al., 2020; Payne, 2017; Duarte, 2020; Li et al., 2020; Sohrabi et al., 2020; Negri et al., 2020; Zimmermann and Curtis, 2020).

Concerning pediatric cases, lower rates of symptomatic progression and lower ratios of hospitalization for the disease were observed compared to the adult population (WHO, 2019; Gorbalenya et al., 2020; Payne, 2017; Duarte, 2020; Li et al., 2020; Sohrabi et al., 2020; Negri et al., 2020; Zimmermann and Curtis, 2020; da Silva Filho et al., 2012; Xiong et al., 2020). In general, there was a lower prevalence in adults, which contributes to the relative scarcity of information regarding pediatric cases of COVID-19 (Zimmermann and Curtis, 2020; da Silva Filho et al., 2012; Xiong et al., 2020). In this way, information about the particularities of COVID-19 in pediatric patients is necessary to aid medical interventions in this population.

\section{Description of Intervention}

The future review will bring together scientific studies that used experimental protocols using a control group and almost experimental ones that did not make use of a control group. It should be emphasized that any study that used interventions with medications, conventional or alternative therapies in relation to the treatment of COVID-19 in children and adolescents will be considered, as shown in Table 2. In all databases, the same descriptors in English and Chinese will be used.

\section{How the Intervention Might Work}

The action of COVID-19 apparently manifests itself more succinctly in the pediatric population (WHO, 2019; Gorbalenya et al., 2020; Payne, 2017; Duarte, 2020; Li et al., 2020; Sohrabi et al., 2020; Negri et al., 2020; Zimmermann and Curtis, 2020;). In this context, it is believed that children naturally have, in their cells, a much smaller amount of coronavirus receptors, which reduces the aggressiveness of the virus (WHO, 2019; Gorbalenya et al., 2020; Payne, 2017; Duarte, 2020; Li et al., 2020; Sohrabi et al., 2020; Negri et al., 2020; Zimmermann and Curtis, 2020; da Silva Filho et al., 2012). Regarding symptoms, when infected children present pathophysiological changes, fever and gastrointestinal alterations present mainly and weeks after contamination, inflammations appear in the vascular system. 9 It has also been identified that they may remain asymptomatic, hindering the identification of the disease and increasing the risks of these individuals being transmitting (WHO, 2019; Gorbalenya et al., 2020; Payne, 2017; Duarte, 2020; Li et al., 2020; Sohrabi et al., 2020; Negri et al., 2020; Zimmermann and Curtis, 2020;).

\section{Why it is Important to do this Review}

The importance of this review is to provide adequate and high-quality information to health professionals who are at the forefront of combating COVID-19, so the information to be included in the future review can be useful to guide attitudes health professionals regarding the prevention of COVID-19 in children and adolescents.

\section{Research Question}

There is a possibility that there will be literary consensus on the forms of treatment and manifestation of COVID-19 in young pediatric patients. Thus, this study protocol aims to provide adequate and reliable support for the production of a systematic review with an overview of the scientific data regarding the pediatric population and COVID-19.

\section{Methods}

\section{Study Design}

The present study is a systematic review protocol based on the recommendations of the PRISMA-P checklist (Moher et al., 2015). In addition, this study is in agreement with the International Medical Journal Editors committee (ICMJE) (ICMJE 2019).

\section{Protocol Registration}

This research is previously registered with the International Prospective Register of Systematic Reviews (PROSPERO) under ID: CRD42020181835.

\section{Criteria for Considering for this Review}

To systematically define the steps of the future systematic review, the scheme described by the Joanna Briggs Institute was adopted, where the structure helps in defining the steps to be followed for the construction of a systematic review (Moola et al., 2015). The method called Population, Intervention and Design ("PID") recommends the prior definition of the study population, the intervention used by the study and the design of the studies that may be part of the review (Tufanaru et al., 2017). In this sense, Table 1 shows the PID of the present study.

\section{Research Strategy}

The literature search will be done purely on the electronic research platforms, having as a filter the date of the works, which will be accepted only if they are 
published from November 2019 to 2020, in addition to the languages selected for the searches, which will be English and Chinese. The databases defined for the search were: PubMed, Google Scholar, LILACS and CINAHL.

\section{Search Methods}

Regarding the methods used in the search for academic articles, we will use the descriptors: Child, Children and COVID-19, both registered on the MeSH platforms. The descriptors will be linked by the Boolean operators OR and AND. It should be noted that the same word grid will be translated into Chinese. In this sense, the terms to be used in the searches will be: COVID-19 AND Child OR Children.

\section{Study Inclusion Criteria}

The inclusion criteria to be considered for the studies are: (i) Scientific text that addresses the theme of covid19 in relation to young pediatric patients aged 6 to 17 years. (ii) The study designs considered will be: Clinical, observational trials, literature review, systematic review, short communications, brief communications and letter to the editor. In all databases, the same descriptors in English and Chinese will be used, as shown in Table 2.

\section{Data Management}

The electronic database used for the development of the research will be the Rayyan ${ }^{\circledR}$ Qatar Computing Research Institute (QCRI) -Web application (open source) -which will filter and store the articles in the literature, whose good functionality occurs in the easy handling and availability on various technological devices (Ouzzani et al., 2016). In this database, the options of the author and collaborators are offered to have access to the systematic review data separately, thus ensuring the organization and mitigation of possible errors during the searches (Ouzzani et al., 2016). To guarantee the "blinding" of the researchers involved, 1 member of the research team will be responsible for managing the database created in Rayyan ${ }^{\circledR}$, having access to all files included by the research team. While the other members will only have access to their individual searches.

\section{Data Sorting and Extraction}

A team composed of 3 researchers will perform the search for scientific texts in electronic databases in a "blind" way. The searches will be divided into three stages:

(1) Studies will be considered for inclusion based on titles. After selection by title, the studies will be implemented in the database created by the research team in the Rayyan ${ }^{\circledR}$ application

(2) Abstracts of the texts selected in step 1 will be read on the Rayyan ${ }^{\circledR}$ platform. After reading, the researchers involved will decide which texts will be directed to step 3. During this process, any conflict regarding the decision of the research team responsible for the screening will be resolved by an external researcher

(3) The texts selected in step 2 will be read in full and the researchers will individually decide which texts will be included. Subsequently, the conflicts of this stage should be resolved through discussion among the researchers involved in the screening, if necessary, another member of the research (not involved with the study selection phase) will be consulted for the resolution of conflicts

\section{Bias Analysis}

The Overview Quality Assessment Questionnaire (OQAQ) will be used to assess the methodological quality of the studies: (i) Systematic review, (ii) Literature review, (iii) Short communication and (iv) Short communication (Oxman and Guyatt, 1991). In the OQAQ, the score for each study evaluated varies from 1 to 9. Thus, the articles will be classified as follows: Poor: Scores <4; Strong: Scores > 5 (Oxman and Guyatt, 1991). Through the quality scale of observational studies developed by Loney, the methodological quality of the observational studies to be included in this research will be analyzed (Loney et al., 1998).

The Loney scale discriminates the degree of risk of bias (low, high or uncertain), for the following observations: Methodological bias; result bias and reproducibility bias (Loney et al., 1998). The quality analyzes of the evidence were carried out separately "blindly" by three researchers. Clinical trials will have their quality analyzed by the Cochrane collaboration scale (De Carvalho et al., 2013). It should be noted that the analysis of biases will be carried out in a "blind" manner by a team of 3 researchers, the analyzes will be carried out individually. After the process, another researcher (Not involved with the bias analysis process) will be responsible for checking the agreement between the bias analysis team and for extracting the final result of the process.

Table 1: Population, Intervention and Design (PID) of the studies to be considered for the future systematic review

\begin{tabular}{ll}
\hline Population & Young pediatric \\
\hline Intervention & No filters \\
Design (study design) & No filters \\
\hline
\end{tabular}

Table 2: Descriptors and synonyms to be used when searching different databases

\begin{tabular}{ll}
\hline Descriptor & Synonyms \\
\hline In English & \\
COVID-19 & Coronavirus disease \\
Child & Children \\
In Chinese & \\
COVID-19 & 冠狀病毒病 \\
孩子 & 孩子們 \\
\hline
\end{tabular}




\section{Summary of Quantitative Data}

The agreement between the researchers in relation to the bias analysis, will be verified by the Interclass Correlation Coefficient (ICC) (For the data of the OQAQ scale) and by the kappa coefficient (for the data of the Loney and Cochrane scales) (Loney et al., 1998; De Carvalho et al., 2013; Miot, 2016). The magnitude considered for the ICC and Kappa results will be: Absence: ICC or Kappa $=\leq 0$; poor: ICC or Kappa $=0-0.19$; weak: ICC or Kappa $=0.20$ 0.39; moderate: ICC or Kappa $=0.30-0.59$; substantial: ICC or Kappa $=0.60-0.79$; and almost complete: ICC or Kappa $=\geq 0.80$ (Miot, 2016).

If it is possible to group the results of the studies to be brought together in this review through meta-analysis, the effects of the component interventions will be measured through the difference in standardized and grouped means and/or by the relative risk, using a random effect model (Lipset and Wilson, 2001). Review restrictions may include excluding insufficient results of interest from metaanalyzes. Thus, if there are at least two studies of adequate power reporting the same result with units of similar measures, they will be included in the meta-analyzes (Turner et al., 2013). In addition, when necessary, the study authors will be contacted to request lost data. The potential impact of missing attrition/result data bias on the result of interest will be assessed based on the Loney, Cochrane and OQAQ bias scales (Oxman and Guyatt, 1991; Loney et al., 1998; De Carvalho et al., 2013).

The heterogeneity of the studies will be analyzed by Cochran's Q and I2 statistics. Studies will be considered heterogeneous when: I2>50\% and significance level $<0.05$ (Dinnes et al., 2005). It is assumed that there will be clinical heterogeneity between the included studies. The inverse variance meta-analytical method will be used (Lipset and Wilson, 2001). Sensitivity analyzes will be conducted by investigating the change in the point estimate if the Metaanalyzes are limited to "low bias" studies and investigating the impact of outlier studies (Lipset and Wilson, 2001).

It should be noted that dichotomous outcomes (i.e., involve multiple variables) will be presented as Risk Ratios (RR) in preference to Odds Ratios (OR). The results of the time until the event will be presented as Risk Rates (HR). Continuous data will be calculated as Weighted Mean Differences (WMD) or Standardized Mean Differences (SMD). Relevant 95\% Confidence Intervals (CIs) will be calculated. The Number Needed to Treat the additional Benefit or damage (NNTB, NNTH) will be calculated as appropriate. All analyzes will be performed using open Source R software (Version 4.0.1, Foundation for Statistical Computing®, Vienna, Austria) and the level of significance considered will be $\mathrm{p}<0.05$.

\section{Synthesis of Qualitative Data}

Qualitative data will be broken down using tables that will contain the main details of each scientific text included in this review. When appropriate, graphics will be used to expose the studies' eligibility procedures and to expose the quality of the analyzed evidence (Egger et al., 1997). A pair of researchers will be assigned to discriminate qualitative data.

\section{Reporting and Dissemination of Results}

The material related to the treatment of COVID-19 in the pediatric population aged 6 to 17 years old, extracted from the literature and compiled in the review, will be of great importance for the scientific community in view of the current moment, in view of the way in which virus manifests in the body of this population. What differs and makes the symptomatology of this population peculiar when compared to other age groups, demonstrating the need for research and reliable scientific survey about the behavior of the disease and ways of treating them.

The perspective of this Protocol is that the future systematic review is finalized and ready for publication by the second semester of 2021, it should be noted that due to the urgency of disseminating information based on scientific evidence on the subject, the findings of the review will be reported to the scientific community and made available for publication, as soon as they are available. In this sense, for the dissemination process, the results may be published in a "pre-press" database before formal peer review.

\section{Discussion}

When considering that there are particularities of the physiological reactions of pediatric patients in relation to COVID-19, it is highlighted that, despite the occurrence of several cases of contamination in children and adolescents, there is no consensus on how to proceed with treatment in this population (WHO, 2019; Payne, 2017; Zimmermann and Curtis, 2020; da Silva Filho et al., 2012). Thus, we aim to provide scientific content, with the expectation of highlighting a crucial point for the prevention of COVID-19 in pediatric patients.

The systematic review research method becomes the most appropriate to bring this answer, because it makes it possible to group scientific data available in the accessible literature. Thus, it will allow the screening of the most relevant findings in relation to the analyzed theme, which makes it possible to minimize errors when making any professional decision based on scientific texts and providing an accurate answer to the scientific community (Allen and Olkin, 1999; Petitti et al., 2009).

In this case, reviews are usually focused on seeking a single study design (i.e., only clinical trials or only observational studies, etc.) in order to reduce methodological biases (Allen and Olkin, 1999). Despite 
the effectiveness of reducing biases by grouping studies that have a similar design, precious data can be lost because they are available in research that has other designs (Higgins et al., 2019). Thus, in order to gather as much information as possible, the systematic review technique called "Overview" is encouraged among researchers (Tufanaru et al., 2017).

The reviews of the type "overview", allow the use of several tools for the analysis of the biases, since the tools are adequate to the designs of the grouped studies (Aromataris and Pearson, 2014). In addition, studies such as literature review can also be included in an overview, which increases the quality of the data synthesis to be made available to readers in relation to a specific theme (Tufanaru et al., 2017; Aromataris and Pearson, 2014; Harris et al., 2014).

Thus, based on the understanding of a systematic review with an overview of this study protocol, it implemented steps to optimize consistency and verify the accuracy of the scientific data to be gathered. Because there are risks when only one reviewer does the screening, extracts and evaluates the studies (Tufanaru et al., 2017; Wright et al., 2007). In addition to this, the analysis tools were anticipated in this protocol, which makes it possible for researchers to become familiar with the tools and procedures beforehand, thus increasing the methodological quality of the future systematic review to be produced based on this study protocol (Tufanaru et al., 2017; Gartlehner et al., 2020).

\section{Acknowledgement}

For your support and encouragement for the development of this academic article, we thank the State University of Ceará (UECE), the Federal University of Rio Grande do Norte (UFRN), the Physical Activity and Health (AFISA) research base, the Child and Adolescent Maturation Research Group (GEPMAC), the Interprofessional Nucleus of Chronic Diseases (NIDOC). The National Council for Scientific Development (CNPQ) and the Higher Education Personnel Improvement Coordination (CAPES).

\section{Author's Contributions}

All authors equally contributed in this work.

\section{References}

Allen, I. E., \& Olkin, I. (1999). Estimating time to conduct a meta-analysis from number of citations retrieved. Jama, 282(7), 634-635. https://jamanetwork.com/journals/jama/articleabstract/2682927
Aromataris, E., \& Pearson, A. (2014). The systematic review: an overview. AJN The American Journal of Nursing, 114(3), 53-58. https://journals.lww.com/ajnonline/FullText/2014/0300 0/The_Systematic_Review_An_Overview.28.aspx

da Silva Filho, L. V. R. F., Zerbinati, R. M., Tateno, A. F., Boas, L. V., De Almeida, M. B., Levi, J. E., ... \& Pannuti, C. S. (2012). The differential clinical impact of human coronavirus species in children with cystic fibrosis. The Journal of Infectious Diseases, 206(3), 384-388. https://academic.oup.com/jid/article/206/3/384/2192 311 ? $\operatorname{login}=$ true

De Carvalho, A., Silva, V., \& Grande, AJ (2013). Bias risk assessment of randomized clinical trials using the Cochrane collaboration tool. Diagn Treatment, 18 (1), 38-44. http://files.bvs.br/upload/S/14139979/2013/v18n1/a3444.pdf

Dinnes, J., Deeks, J., Kirby, J., \& Roderick, P. (2005). A methodological review of how heterogeneity has been examined in systematic reviews of diagnostic test accuracy. Health Technology Assessment (Winchester, England), 9(12), 1-113. https://europepmc.org/article/nbk/nbk62298

Duarte, P. M. (2020). COVID-19: Origin of the new coronavirus. Brazilian Journal of Health Review, 3 (2), 3585-3590. https://www.brazilianjournals.com/index.php/BJHR/ article/view/9131

Egger, M., Smith, G. D., \& Phillips, A. N. (1997). Metaanalysis: principles and procedures. BMJ, 315(7121), 1533-1537. https://www.bmj.com/content/315/7121/1533.full

Gartlehner, G., Affengruber, L., Titscher, V., Noel-Storr, A., Dooley, G., Ballarini, N., \& König, F. (2020). Single-reviewer abstract screening missed 13 percent of relevant studies: a crowd-based, randomized controlled trial. Journal of Clinical Epidemiology, 121, 20-28. https://www.sciencedirect.com/science/article/pii/S0 895435619309825

Gorbalenya, A. E., Baker, S. C., Baric, R., Groot, R. J. D., Drosten, C., Gulyaeva, A. A., ... \& Ziebuhr, J. (2020). Severe acute respiratory syndrome-related coronavirus: The species and its viruses-a statement of the Coronavirus Study Group. https://digital.csic.es/handle/10261/212994

Harris, J. D., Quatman, C. E., Manring, M. M., Siston, R. A., \& Flanigan, D. C. (2014). How to write a systematic review. The American Journal of Sports Medicine, 42(11), 2761-2768. https://journals.sagepub.com/doi/abs/10.1177/03635 46513497567 
Higgins, J. P., Thomas, J., Chandler, J., Cumpston, M. Li, T., Page, M. J., \& Welch, V. A. (Eds.). (2019). Cochrane handbook for systematic reviews of Interventions. John Wiley \& Sons.

ICMJE. (2019) Website. International Committee of Medical Journal Editors http://www.icmje.org

Li, Q., Guan, X., Wu, P., Wang, X., Zhou, L., Tong, Y., ... \& Feng, Z. (2020). Early transmission dynamics in Wuhan, China, of novel coronavirusinfected pneumonia. New England Journal of Medicine.

https://www.nejm.org/doi/full/10.1056/NEJMOa2 001316

Lipset, M. W., \& Wilson, D. B. (2001). Practical metaanalysis, Applied social Research Methods Series.

Loney, P. L., Chambers, L. W., Bennett, K. J., Roberts, J. G., \& Stratford, P. W. (1998). Critical appraisal of the health research literature: prevalence or incidence of a Health Problem. Chronic Dis Can, 19(4), 170-6.

Miot, H. A. (2016). Agreement analysis in clinical and experimental trials. J Vasc Bras, 15(2), 89-92. https://www.redalyc.org/pdf/2450/245046412001 2.pdf

Moher, D., Shamseer, L., Clarke, M., Ghersi, D., Liberati, A., Petticrew, M., Shekelle, P., \& Stewart, L. A. (2015). Itens de Relatório Preferidos para Declaração Sistemática e Declaração de Protocolos de Meta-Análise (PRISMA). Revise System, 2015; 4 (1); 1. [In Portuguese, English abstract].

Moola, S., Munn, Z., Sears, K., Sfetcu, R., Currie, M., Lisy, K., ... \& Mu, P. (2015). Conducting systematic reviews of association (etiology): the Joanna Briggs Institute's approach. JBI Evidence Implementation, 13(3),

163-169.

https://journals.lww.com/ijebh/fulltext/2015/09000/con ducting_systematic_reviews_of_association.8.aspx

Negri, E. M., Piloto, B. M., Morinaga, L. K., Jardim, C. V. P., Lamy, S. A. E. D., Ferreira, M. A., ... \& Deheinzelin, D. (2020). Heparin therapy improving hypoxia in COVID-19 patients-a case series. Frontiers in Physiology, 11. https://www.ncbi.nlm.nih.gov/pmc/articles/PMC7 604350/

Ouzzani, M., Hammady, H., Fedorowicz, Z., \& Elmagarmid, A. (2016). Rayyan-a web and mobile app for systematic reviews. Systematic Reviews, 5(1), 110. https://link.springer.com/article/10.1186/s13643016-0384-4

Oxman, A. D., \& Guyatt, G. H. (1991). Validation of an index of the quality of review articles. Journal of Clinical Epidemiology, 44(11), 1271-1278. https://www.sciencedirect.com/science/article/abs/pi i/089543569190160B
Payne, S. (2017). Family Coronaviridae. Viruses, 149. https://www.ncbi.nlm.nih.gov/pmc/articles/PMC714 9805/

Petitti, D. B., Teutsch, S. M., Barton, M. B., Sawaya, G. F., Ockene, J. K., \& DeWitt, T. (2009). Update on the methods of the US Preventive Services Task Force: insufficient evidence. Annals of Internal Medicine, 150(3), 199-205. https://www.acpjournals.org/doi/full/10.7326/00034819-150-3-200902030-00010

Sohrabi, C., Alsafi, Z., O'Neill, N., Khan, M., Kerwan, A., Al-Jabir, A., ... \& Agha, R. (2020). World Health Organization declares global emergency: A review of the 2019 novel coronavirus (COVID-19). International Journal of Surgery, 76, 71-76. https://www.sciencedirect.com/science/article/pii/S1 743919120301977

Tufanaru, C., Munn, Z., Aromataris, E., Campbell, J., \& Hopp, L. (2017). Systematic reviews of effectiveness. Joanna Briggs Institute Reviewer's Manual, 3.

Turner, R. M., Bird, S. M., \& Higgins, J. P. (2013). The impact of study size on meta-analyses: examination of underpowered studies in Cochrane reviews. PloS One, 8(3), e59202. https://journals.plos.org/plosone/article?id=10.1371/ journal.pone.0059202

WHO. (2019). World Health Organization. Coronavirus disease 2019 (COVID-19) Situation Report - 51. Website. https://www.who.int/docs/defaultsource/coronaviruse/situation-reports/20200311sitrep-51-Covid-19.pdf?sfvrsn=1ba62e57_10. Published 2019. Acessed June 20, 2020.

Wright, R. W., Brand, R. A., Dunn, W., \& Spindler, K. P. (2007). How to write a systematic review. Clinical Orthopaedics and Related Research®, 455, 23-29. https://journals.lww.com/clinorthop/fulltext/2007/02 000/how_to_write_a_systematic_review.7.aspx

Xiong, X., Chua, G. T., Chi, S., Kwan, M. Y. W., Wong, W. H. S., Zhou, A., ... \& Ip, P. (2020). A comparison between chinese children infected with coronavirus disease-2019 and with severe acute respiratory syndrome 2003. The Journal of Pediatrics, 224, 30-36. https://www.sciencedirect.com/science/article/abs/pii/S 0022347620307435

Zimmermann, P., \& Curtis, N. (2020). Coronavirus infections in children including COVID-19: an overview of the epidemiology, clinical features, diagnosis, treatment and prevention options in children. The Pediatric Infectious Disease Journal, 39(5), 355 https://www.ncbi.nlm.nih.gov/pmc/articles/PMC715 $8880 /$ 